\title{
Surface enhanced infrared absorption of nucleic acids on gold substrate
}

\author{
G.I. Dovbeshko1, V.I. Chegel$^{2}$, N.Y. Gridina ${ }^{3}$, O.P. Repnytska', Y.M. Shirshov², V.P. Tryndiak ${ }^{4}$, \\ I.M. Todor ${ }^{4}$, S.A.Zynio ${ }^{2}$ \\ 1-Institute of Physics of National Academy of Sciences of Ukraine, Prospect Nauki 46, Kyiv, 03039, Ukraine, \\ galyna@ukma.kiev.ua; \\ 2-Institute of Semiconductor Physics of the National Academy of Sciences of Ukraine, Prospect Nauki 45, Kiev, 03039, Ukraine; \\ 3-A.P. Romodanov Institute of Neurosurgery of Medical Academy of Sciences of Ukraine, 32 Manuilskogo str., Kiev-04050; \\ 4-Kavetsky Institute of Experimental Pathology, Oncology and Radiobiology, Vasylkivska 45, Kyiv, 03022, Ukraine.
}

\begin{abstract}
Data on surface enhanced infrared absorption (SEIRA) of nucleic acids deposited on the metal surface have been obtained in the experiment in FTIR reflectance mode. As metal

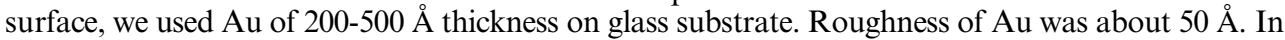
our experimental conditions, the enhancement factor of SEIRA was 3 to 5. We obtained different enhancement factors for different vibrations of nucleic acids. Application of this method to tumour nucleic acid gave a possibility to reveal some structural peculiarities of their sugar-phosphate backbone and those after application of anti-cancer drugs.
\end{abstract}

Keywords: surface enhanced infrared absorption (SEIRA), RNA, DNA, resistant cancer, sensitive cancer, gold substrate, FTIR reflectance, anti-cancer drugs.

Paper received 13.04.01; revised manuscript received 20.06.01; accepted for publication 13.07.01.

\section{Introduction}

The effect of surface enhanced infrared absorption (SEIRA) is well known during last 20 years [1]. It consists in the essential increase (by factor $\sim 10^{2}$ ) of the intensities of some IR bands of the molecules adsorbed on metal particles or surfaces. It can be accompanied by increase of intensities of some bands or even decrease of intensities of other bands (which can be characterized as «selection rules»). This effect has been registered first by Hartstein et al. in 1980 [2], similarly to the SERS effect, however only recently it was named as SEIRA and got practical application in the last decade [3-7], mainly due to intensive study of biological molecules [6]. An enhancement factor, which could be reached with SEIRA, equals to $100 \ldots 1000$. The interpretation of this effect similar to more known SERS (surface enhanced Raman spectra) effect includes at least two mechanisms such as local enhancement of external electric field near the rough metal surface due to excitation of surface plasmons and specific increase of the polarizability of the molecules at their adsorption on metal surface [8].

\section{Methods and materials}

FTIR methods, when applied for structural studies of biological molecules, have essential sensitivity; however, in modern genetic and biochemical applications, where only very small amounts of materials are available, there is a necessity to have increased sensitivity. For this reason, any enhancement of the signal is very important. Nucleic acids belong to the materials whose isolation requires expensive materials and time, so enhancing the FTIR absorption even by factor 3-5 gives one a possibility to enhance both sensitivity and accuracy of the analytical methods. An application of metal rough surface as substrate for studying absorbed molecules could lead to enhancement of the FTIR signal in reflectance or attenuated total reflectance modes. The enhanced FTIR signals from vibrations of nucleic acids deposited on noble metal surface have been registered in the experiment with reflectance attachment (Fig.1). As metal surface, we used $\mathrm{Au}$ of 200 to $500 \AA \AA$ thickness on glass substrate. Roughness of $\mathrm{Au}$ was not greater than $50 \AA$. The samples of genomic DNA and total fraction of RNA isolated from 


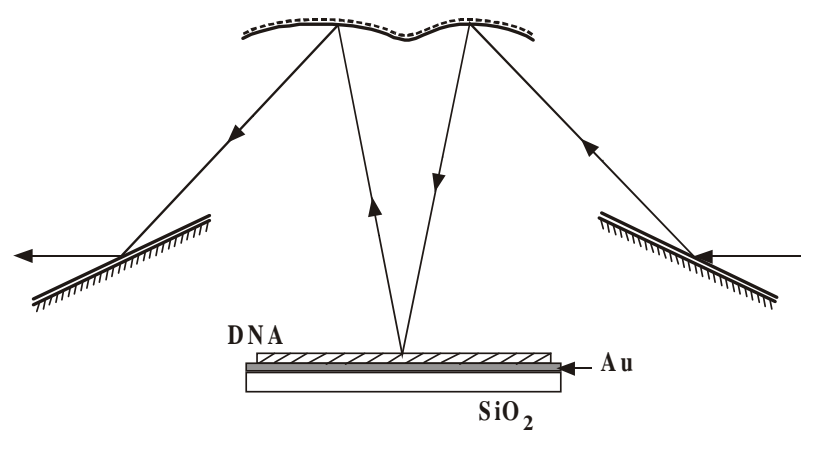

Fig. 1. Geometry of SEIRA experiment.

human brain tumours of different stages of malignancy astrocytoma (II grade of malignancy) and glioblastoma (IY grade of malignancy) as well as of total fraction of DNA from Carcinoma Guerina cells of Wistar line rats. Changes in the structure of DNA caused by application of cisplatin and doxorubicin as anticancer drugs to these two types of cancers were a subject of investigation, too. The DNA was isolated from cancer cells of rats exposed by $9.5 \mathrm{mG} / \mathrm{kG}$ doxorubicin or $6 \mathrm{mG} / \mathrm{kG}$ cisplatin during
1 hour. The samples of DNA from aqueous solution were deposited on the Au surface of 200-500 A thickness and then were liophilically dried. All samples were kept in closed boxes with $\mathrm{P}_{2} \mathrm{O}_{5}$ water adsorbent. Nucleic acid concentration was about $1 \mu \mathrm{g} / \mu \mathrm{l}$. As reference DNA and RNA we used the total fraction of nucleic acids isolated from the same type of normal tissue of Wistar rats.

The reflectance attachment used in the experiment has the light incidence angle close to $16.5^{\circ}$. The FTIR spectra were registered in the $380-5300 \mathrm{~cm}^{-1}$ region with IFS 48 Bruker instrument. Deconvolution and decomposition of the bands has been performed with Opus-2.2. program. The positions of the bands have been estimated using the method of second derivative. The enhancement factor of SEIRA for different vibrations was calculated as ratio of integral areas of corresponding bands in SEIRA and conventional geometry (Table 1).

The microphotograph of the Au surface used as substrate for SEIRA was obtained by atomic force microscope (AFM). We used tapping mode of AFM imaging, using a commercial Nanoscope IIIa (Digital Instrument, Santa Barbara, CA). Scans were performed in the tapping force mode using commercially available AFM tips (made of silicon nitride). Scanning frequency was approximately $1 \mathrm{~Hz}$ in all experiments.

Table 1. The SEIRA enhancement factor of different vibrations for nucleic acid.

\begin{tabular}{|c|c|c|c|c|c|c|c|}
\hline Reference & \multicolumn{2}{|c|}{$\mathrm{Au}, 200 \mathrm{~A}^{\circ}$} & \multicolumn{2}{|c|}{$\mathrm{Au}, 300 \mathrm{~A}^{\circ}$} & \multicolumn{2}{|c|}{$\mathrm{Au}, 400 \mathrm{~A}^{\circ}$} & \multirow[t]{2}{*}{ Band assignment } \\
\hline $\begin{array}{l}\text { Band } \\
\text { position, } \\
\mathrm{cm}^{-1}\end{array}$ & $\begin{array}{l}\text { Band } \\
\text { position, } \\
\mathrm{cm}^{-1}\end{array}$ & $\begin{array}{l}\text { Intensity } \\
\text { enhance- } \\
\text { ment factor }\end{array}$ & $\begin{array}{l}\text { Band } \\
\text { position } \\
\mathrm{cm}^{-1}\end{array}$ & $\begin{array}{l}\text { Intensity } \\
\text { enhance- } \\
\text { ment factor }\end{array}$ & $\begin{array}{l}\text { Band } \\
\text { position, } \\
\mathrm{cm}^{-1}\end{array}$ & $\begin{array}{l}\text { Intensity } \\
\text { enhance- } \\
\text { ment factor }\end{array}$ & \\
\hline 3350 & 3359 & 4.3 & 3354 & 2.0 & 3362 & 1.3 & $\mathrm{O}-\mathrm{H}, \mathrm{N}-\mathrm{H}, \mathrm{C}-\mathrm{H}$ \\
\hline 1655 & 1653 & 2.9 & 1649 & 1.4 & 1654 & 1.3 & $\begin{array}{l}\mathrm{C}=\mathrm{O}, \mathrm{C}=\mathrm{N}, \mathrm{N}-\mathrm{H} \text { of } \\
\text { Adenine, Thymine, } \\
\text { Guanine, Cytosine }\end{array}$ \\
\hline 1528 & 1530 & 3.0 & 1529 & 1.1 & 1529 & 1.2 & $\begin{array}{l}\mathrm{C}=\mathrm{N} \text { Adenine, } \\
\text { Cytosine }\end{array}$ \\
\hline 1490 & 1486 & 2.6 & 1489 & 1.0 & 1488 & 1.1 & Def. C-H \\
\hline 1419 & 1418 & 2.8 & 1418 & 1.0 & 1418 & 1.1 & Def. C-H \\
\hline 1373 & 1370 & 3.8 & 1371 & 1.6 & 1371 & 1.5 & Def. N-H, C-H \\
\hline 1238 & 1230 & 2.4 & 1230 & 1.0 & 1230 & 1,1 & Str. $\mathrm{PO}_{2}^{-}$asym \\
\hline 1089 & 1084 & 2.1 & 1084 & 0.8 & 1084 & 0.9 & Str. $\mathrm{PO}_{2}^{-}$sym \\
\hline 964 & 963 & 2.4 & 964 & 0.9 & 963 & 1.1 & $\begin{array}{l}\text { C-C,C-O } \\
\text { deoxyribose }\end{array}$ \\
\hline 892 & 889 & 1.7 & 892 & 0.9 & 889 & 0.6 & $\begin{array}{l}\text { C-C, C-O } \\
\text { deoxyribose }\end{array}$ \\
\hline 833 & 831 & 2.0 & 829 & 0.8 & 831 & 1.3 & $\begin{array}{l}\mathrm{C}_{2}, \text { - endo confor- } \\
\text { mation of shugar }\end{array}$ \\
\hline
\end{tabular}




\subsection{SEIRA experiment}

The adduced below theoretical interpretation of the effect is connected with enhancement of external electric field due to excitation of local (surface) plasmon vibrations at essential curvature of rough surface or on metal particles. In such systems, according to [8], the electric field in the point of space $r$ could be written as follows (all formulae presented here are taken from [8]) :

$$
E(r, t)=\exp (-i \omega t)\left[E_{0}(\omega)+E_{1}(r, \omega)\right]
$$

Here $\omega$ is frequency, $t$ is time. The additional term $E_{1}$ $(r, \omega)$ connected with excitation of local (surface) plasmon vibrations. The amplification of the external field can be presented as

$E_{\alpha}(r, \omega)=g_{\alpha \beta}(r, \omega) E_{0 \beta}(\omega)$,

where $g_{\alpha \beta}(r, \omega)$ is a coefficient of amplification.

For the matrix elements of dipole moment of the transition between the states $i$ (with energy $\mathrm{e}_{i}$ ) and $f$ for the adsorbed molecule of nucleic acid, we have

$<f|d \alpha(r)| i>=h_{\alpha \beta}{ }^{(f i)}(r)<f\left|d_{0, \beta}\right| i>$,

where $<f\left|d_{0}\right| i>$ is the matrix element of dipole moment of transition for isolated molecule.

According to classical approximation, the matrix elements of the Hamiltonian of the interaction of the adsorbed molecule with field are:

$<f|H(r, \omega) i>=-<f| d_{\alpha}(r) \mid i>E_{\alpha}(r, \omega)=$

$=-h_{\alpha \beta}{ }^{(f i)}(r) g_{\alpha \gamma}(r, \omega)<f\left|d_{0, \beta}\right| i>E_{0, \gamma}(\omega)$

Having performed substitution from Eq. (4) into the expression

$\omega_{f i}(r, \omega)=(2 p / \hbar)|<f| H(r, \omega)|i>|^{2} \delta\left(h \omega-\varepsilon_{f}+\varepsilon_{i}\right)$

for the rate of the $i \rightarrow f$ transition, multiplied the result by the energy $h \omega$ of absorbed quantum, and divided by the density of the incident energy flow $c E_{0}^{2} / 8 \pi$ (where $c$ - the light velocity), we obtain the expression for the effective cross-section of light absorbance by adsorbed molecule:

$\sigma_{\alpha}(r, \omega)=\frac{16 \pi^{2} \omega_{f i}}{c E_{0}^{2}}|<f| H(r, \omega) \times$

$\times|i>|^{2} \delta\left[h\left(\omega-\omega_{f i}\right)\right] \sim \sigma_{\alpha}^{(0)}|g(r, \omega)|^{2}|h(r)|^{2}$,

where $\sigma_{\alpha}{ }^{(0)}$ is cross-section for the case when metal is absent $\left(\mathbf{d}=\mathbf{d}_{0}, \mathbf{E}=\mathbf{E}_{0}\right)$. The right hand part of the expression (5) is the estimation of the cross-section as to its order of magnitude; this all formulae presented here are taken from [8] estimation shows the dependence of the crosssection on the (averaged over tensor indices) coefficients of amplification of the electric field $g$ and dipole moment $h$ of the transition near the metal surface. The influence of the last factor is probably not essential in some experi-

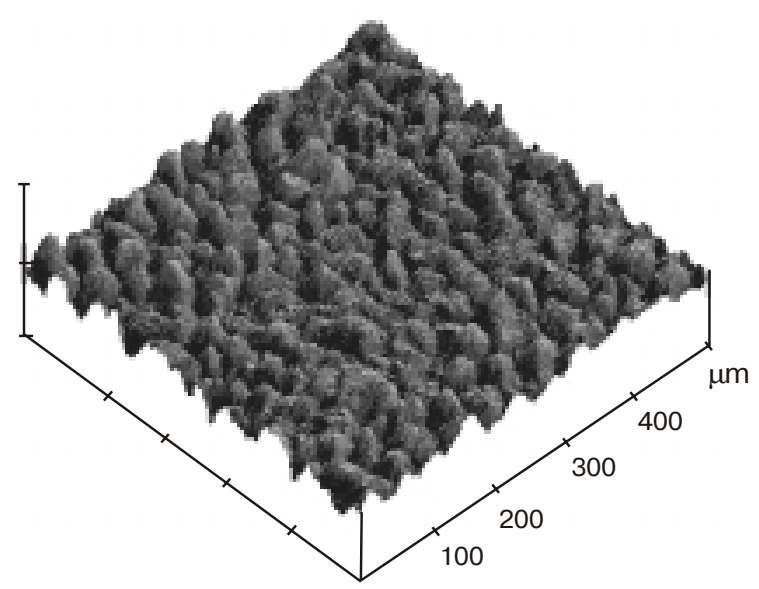

Fig. 2 AFM image of the gold surface used in SEIRA experiment. The size of roughness of the surface is $50 \AA$.

ments where the moments of transition for the absorbed molecules remain unchanged. In the most cases amplification of the electric field $g^{2}$ could be expressed by the proportional to $\varepsilon^{\prime} / \varepsilon^{\prime \prime}$ of the metal surface [8].

The estimation shows that the enhancement of the effect is increased in the case when the characteristic size of roughness of the surface is close to $50-100 \AA$. In our experiment, we used gold substrate with similar size of roughness (Fig. 2) and obtained enhancement of SEIRA by factor 3-5 for different vibrations (Fig. 3). We assume that this is not maximal enhancement, which could be obtained in the system.

\section{Results and discussion}

In the spectra of DNA and RNA, we have two wide bands near 1090 (Phosphate I) and $1230 \mathrm{~cm}^{-1}$ (Phosphate II)

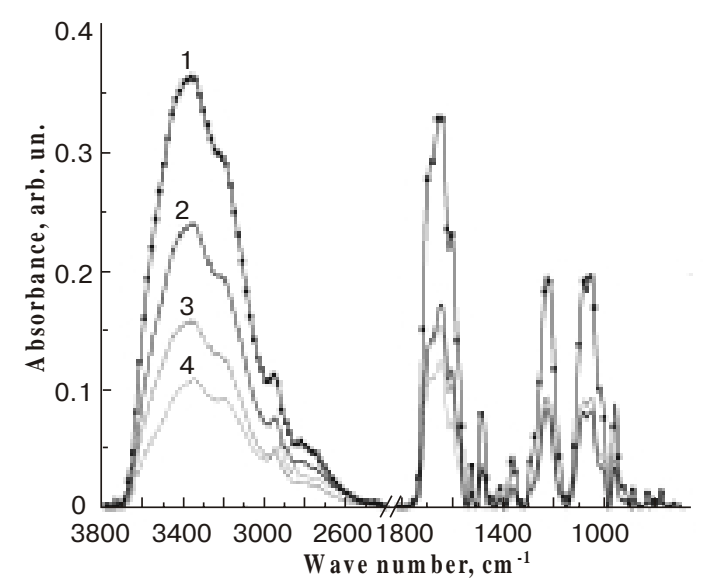

Fig. 3. SEIRA spectra of DNA: 1 - on gold substrate with $200 \AA$ thickness; 2 - $300 \AA, 3-400 \AA, 4-$ on $\mathrm{CaF}_{2}$ substrate. 


\section{G.I. Dovbeshko et al.: Surface enhanced infrared absorption of nucleic acids ...}

attributed to vibrations of sugar-phosphate backbone. Each of these bands consists of several overlapped bands. Decomposition enables one to obtain the positions and intensities of these bands. Earlier [9] we studied RNA isolated from rats (normal and with tumours) and found a number of features specific for tumour RNA. In [9] we have registered the FTIR absorbance spectra of tumour brain nucleic acids on $\mathrm{CaF}_{2}$ and $\mathrm{Si}$ substrates, so we had no possibility for studies of Phosphate I and sugar vibrations in the $700-900 \mathrm{~cm}^{-1}$ region due to restricted transmittance of these substrates. The application of SEIRA to nucleic acids on $\mathrm{Au} / \mathrm{SiO}_{2}$ substrate gave the possibility to obtain good quality spectra in $1100-600 \mathrm{~cm}^{-1}$ region with enhancement factor 3-5 and to reveal additional features of molecular structure of tumour nucleic acids (Fig. 4). In the FTIR spectra of tumour RNA on $\mathrm{Au} / \mathrm{SiO}_{2}$, we found appearance of the band at $1147-1155 \mathrm{~cm}^{-1}$ (Fig. 5, curves $2,3,4$ ) with increasing intensity in the case of more malignant tumour. In the absorption spectra of reference RNA, this band is practically absent. The halfwidth of Phosphate I band was drastically greater in RNA from tumour cells (Fig. 5, curve 2,3,4) in comparison with those for reference RNA. The redistribution of intensities of main peaks of Phosphate 2 band (near 1243 and 1223 for reference RNA) and high frequency shift of the peaks in the case of the most malignant tumour (Figs 4,5 curve 2) have been observed in tumour RNA. More vibration states were calculated under the decomposition of these phosphate bands in the case of tumour RNA as well as DNA. The peculiarities of tumour DNA looks like as RNA with less prominent manifestation.

In the region of sugar moieties of tumour RNA, we have registered not only north-type $\left(\mathrm{C}_{3}\right.$,endo $)$ sugar conformation, but south-type $\left(\mathrm{C}_{2}\right.$,endo), too. Assignment of sugar conformations was made according to $[10,11]$. For

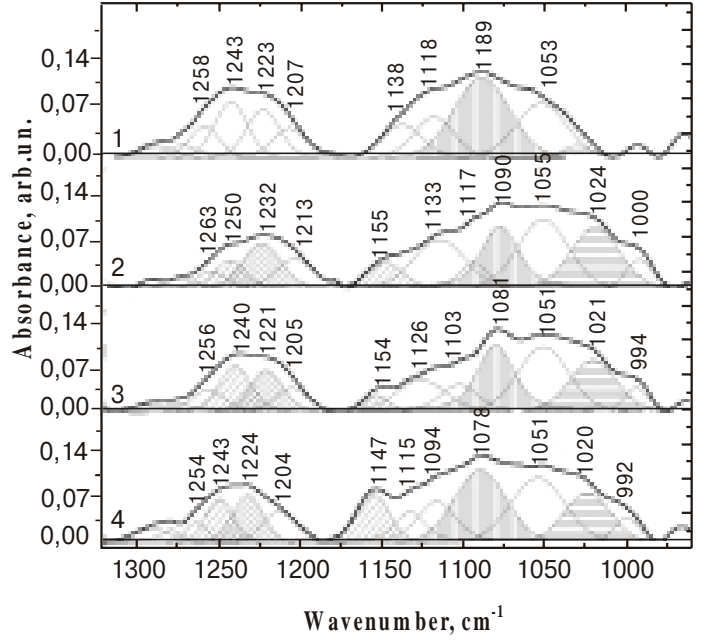

Fig. 5. SEIRA spectra of RNA on the Au substrate: 1 - reference, 2 - glioblastoma (IY grade) 3, 4 - astrocytoma (II grade) in the $960-1320 \mathrm{~cm}^{-1}$ region.

glioblastoma (Fig. 4, curve 2), we found both these conformations with approximately equal intensities. In other tumour RNA (Fig. 4, curve 3, 4), we have registered suppression of any sugar vibrations in the $700-900 \mathrm{~cm}^{-1} \mathrm{re}-$ gion. Arising of the band at the $793 \mathrm{~cm}^{-1}$ (Fig. 4, curve 4) could be attribute to guanine in $\mathrm{C}_{3}$,endo/syn conformation, which appears in Z-conformation of DNA [12].

Under the application of doxorubicin to the sensitive cancer, the structure of DNA in the sugar region looks like that for DNA from normal tissue (Fig. 6). In the case of doxorubicin application, the structure of sugars practically does not change in the resistant cancer cells. A ratio of intensities of Phosphate I and Phosphate II bands

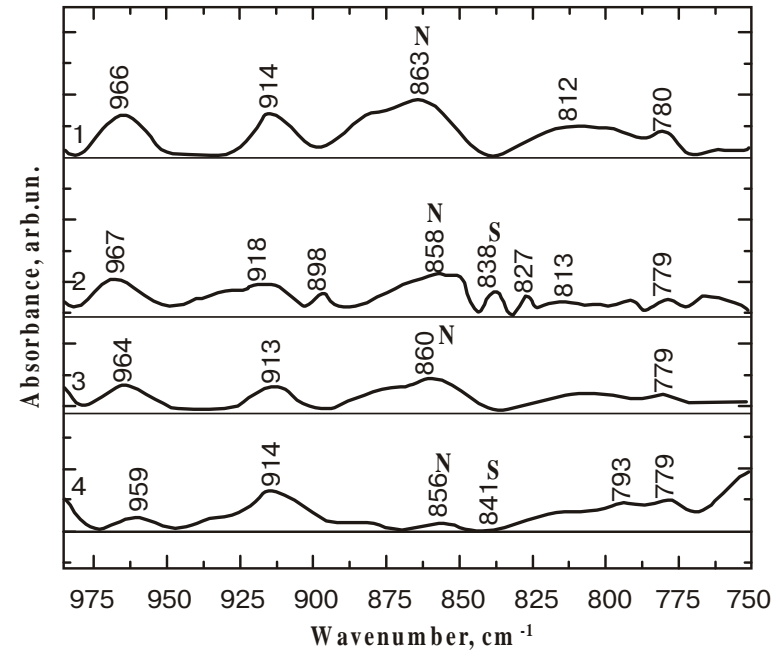

Fig. 4. SEIRA spectra of RNA on the Au substrate: 1 - reference, 2 - glioblastoma (IY grade.) 3, 4 - astrocytoma (II grade) in the 750-970 $\mathrm{cm}^{-1}$ region.

$S Q O, 4(3), 2001$

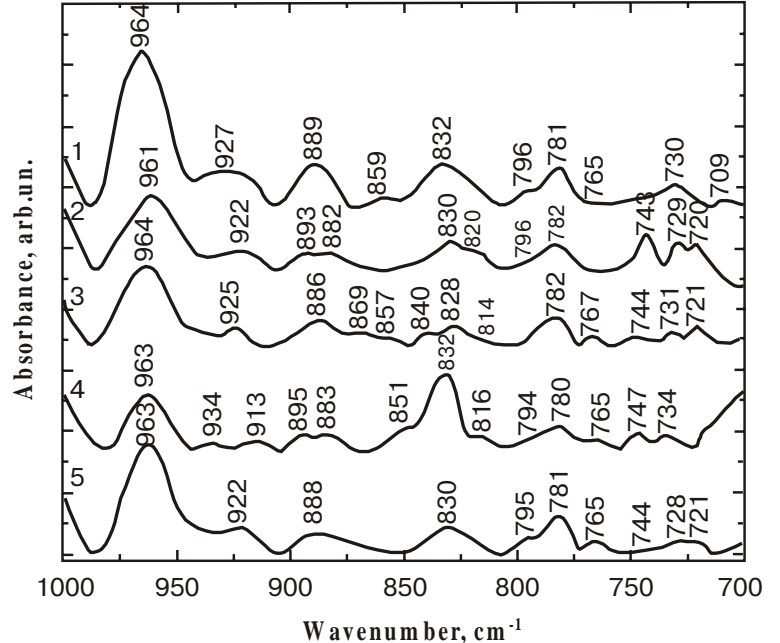

Fig. 6. SEIRA spectra of DNA: 1 - reference spectra, 2 - from resistant tissue, 3 - from resistant tissue after treatment with doxorubicine, 4 - from sensitive tissue, 5 - from sensitive tissue after treatment with doxorubicine. 


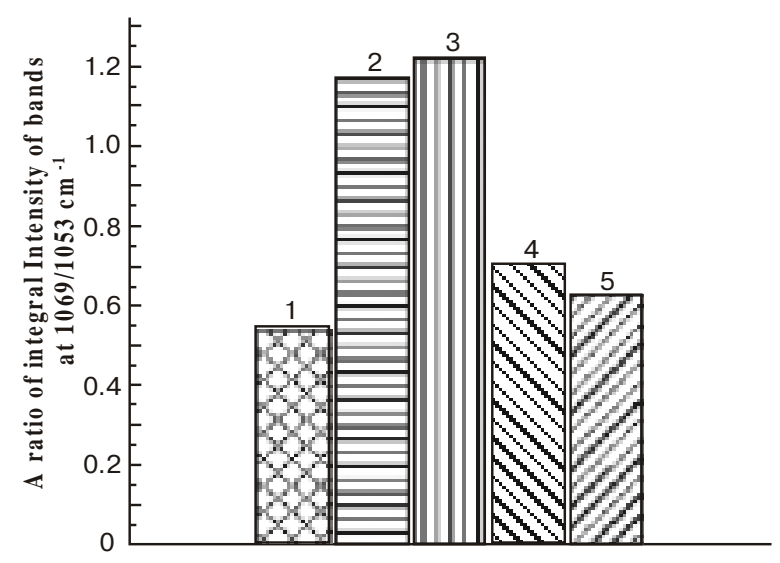

Fig. 7. A ratio of integral intensity of bands at $1069 \mathrm{~cm}^{-1}$ to $1053 \mathrm{~cm}^{-1}$ for DNA: 1 - reference spectra, 2 - from resistant tissue, 3 - from resistant tissue after treatment with doxorubicine, 4 - from sensitive tissue, 5 - from sensitive tissue after treatment with doxorubicine.

in the resistant cancers does not change after treatment by drugs, but in the case of sensitive tumours, this ratio changes by factor 1.5 or more. In the region of OH-NH$\mathrm{CH}$ stretching vibrations, strong $\mathrm{H}$-bonded $\mathrm{NH}$ vibration band near $2600 \mathrm{~cm}^{-1}$ becomes stronger for the resistant cancers after treatment. The spectrum of resistant tumour DNA looks like the spectrum of DNA from normal tissue, and their changes after application of anti tumour drugs are negligibly small. Thus, the DNA of resistant tumour cells could be characterised as rigid structure. The structure of sensitive cancer DNA seems to be flexible, and after application of anti-cancer drugs it drastically changes and becomes close to the structure of DNA from normal tissues. The Fig. 6 confirm abovementioned influence of anti-cancer drugs on the structure of sensitive and resistant cancers in the Phospate 1 band for two different $\mathrm{C}-\mathrm{O}$ vibrations of deoxyribose at $1053 \mathrm{~cm}^{-1}$ (ordering structure of DNA) and $1069 \mathrm{~cm}^{-1}$ (disordering structure of DNA). Calculating the ratio of integral intensity of contributions of these two vibrations, we can characterize the level of disorder in the DNA structure. We concluded about increasing of disorder in resistant cancers in comparison with sensitive cancers. For DNA from sensitive cancer after treatment, the disorder became less and for resistant cancers it became more after treatment by anti-cancer drugs.

\section{Conclusions}

Application of SEIRA to nucleic acids on gold substrate with 200-500 ̊ thickness and size of roughness $50 \AA$ has shown amplification of absorbance by a factor of 3 to 5 for various vibrational modes of nucleic acids. SEIRA has revealed the structural features of the tumour nucleic acid and those after application of anti-cancer drugs connected with conformations of sugars and phosphate groups in the sugar-phosphate backbone.

\section{References}

1. Chang R.K. and Furtak T.E. (Eds.), Surface Enhanced Raman Scattering, Plenum Press, New York - London (1982).

2. Harstain A., Kirtley J.R., Tsang J.C. Infrared Absorption of the Molecular Monolayers with Thin Metal Overlayers // Phys.Rev.Lett. 45, pp. 201-209(1980).

3. Masatoshi Osawa, Masahiko Ikeda, Surface-Enhanced Infrared Absorption of p-Nitrobenzoic Acid Deposited on Silver Island Films: Contributions of Electromagnetic and Chemical Mechanisms // J. Phys. Chem. 95, pp.9914-9919 (1991).

4. Ken-ichi Ataka, Takao Yotsuyanagi, Masatoshi Osawa, Potential-Dependent Reorientation of Water Molecules at an Electrode/Electrolyte Interface Studied by Surface-Enhanced Infrared Absorption Spectroscopy// J. Phys. Chem. 100, pp. 10664-10672 (1996).

5. H.D. Wanzenbцck, B. Mizaikoff, N. Weissenbacher, R. Kellner, Surface Enhanced Infrared Absorption Spectroscopy (SEIRA) using external reflection on low-cost substrates // Fresenius J. Anal. Chem. 362, pp. 15-20 (1998).

6. Ch. Kuhne, G. Steiner, W.B. Fischer, R. Salzer, Surface Enhanced FTIR Spectroscopy on membranes // Fresenius J. Anal. Chem. 360, pp. 750-754 (1998).

7. A. Roseler, E.H. Korte. Surface enhanced infared absorption observed with attenuated total reflection (ATR-SEIRA): modeling the optical responce // Fresenius J.Anal. Chem. 362, pp.51-57(1998).

8. V.A. Kosobukin. Effect of enhancement of external electric field near metal surface and its manifestation in spectroscopy // Surface.Physics, chemistry, mechanics. 12, pp.5-20 (1983).

9. G.I. Dovbeshko, N.Ya. Gridina, E.B. Kruglova, O.P. Paschuk, FTIR spectroscopy studies of nucleic acids damage// Talanta. 53, pp.233-246 (2000).

10. B. Schrader, Infrared and Raman Spectroscopy, WeinheimNew York-Basel-Cambridge-Tokio (1995).

11. E. Taillandier, J. Liquier, and J.A. Taboury in R. J. H. Clarc, R.E. Hester (Eds), Advances in Infrared and Raman Spectroscopy, Wiley Heyden ( 1985).

12. H.A. Tajmir-Riahi, J.F. Neault, M. Naoui, Does DNA acid fixation produce left-handed Z structure? // FEBS Letters, 370, pp.105-108 (1995). 